\title{
Stereopsis Outweighs Gravity in the Control of the Eyes
}

\author{
Hubert Misslisch, ${ }^{1}$ Douglas Tweed, ${ }^{2}$ and Bernhard J. M. Hess ${ }^{1}$ \\ ${ }^{1}$ Department of Neurology, University of Zurich, $\mathrm{CH}-8091$ Zurich, Switzerland, and ${ }^{2}$ Departments of Physiology and \\ Medicine, University of Toronto, Toronto, M5S 1A8, Canada
}

The eyes are controlled by multiple brain circuits, some phylogenetically old and some new, whose aims may conflict. Old otolith reflexes counterroll the eyes when the head tilts relative to gravity. Newer vergence mechanisms coordinate the eyes to aid stereoptic vision. We show that counterroll hinders stereopsis, weakly when you look into the distance but strongly when you look near. The resolution of this conflict is that counterroll virtually vanishes when monkeys look close, i.e., stereopsis overrides gravity-driven reflexes but only on near gaze. This balance between gyroscopic and stereoptic mech- anisms explains many other puzzling features of primate gaze control, such as the weakness of our otolith-ocular reflexes even during far viewing and the strange geometry of the primate counterpitch reflex, which rolls the eyes clockwise when monkeys look leftward while their heads are tipped nose up, but rolls them counterclockwise when the monkeys look rightward, and reverses this pattern when the head is tipped nose down.

Key words: stereopsis; vergence; otolith-ocular; vestibuloocular; ocular torsion; vision; 3-D eye movements
Eye movements are controlled by several neural systems whose aims sometimes conflict. One system, the otolith-ocular reflex, senses the direction of gravity and, among other actions, rolls the eyes toward upright when the head is stationary in a tilted position, a response known as counterroll (Hunter, 1786; Nagel, 1868). When you hold your head $90^{\circ}$ clockwise, or right ear down, for instance, your eyes tilt some $5-10^{\circ}$ counterclockwise in their sockets (Collewijn et al., 1985; Crawford and Vilis, 1991; Haslwanter et al., 1992). This tilt is far too small to hold the eye stable in space, but perhaps it was larger in some distant ancestor of ours, in whom it kept the eyes aligned with the horizon (Carpenter, 1988). In lateral-eyed animals, such as rabbits, the reflex is still strong; a rabbit tilted $90^{\circ}$ counterrolls its eyes $\sim 50^{\circ}$ (van der Hoeve and de Kleijn, 1917; Fleisch, 1922).

Another motor system, vergence, crosses the eyes to look close and uncrosses them to look into the distance, always keeping both lines of sight trained on some single object, the fixation target. Its major function is to facilitate binocular depth vision, or stereopsis, in which the brain uses the small disparities between the images in the two eyes to compute the depths of objects relative to the fixation target (Wheatstone, 1838; von Helmholtz, 1867). Vergence is a phylogenetically new system, weaker in rabbits and even in cats than it is in primates (Hughes, 1972; Zuidam and Collewijn, 1979).

Geometry brings vergence and otolith reflexes into conflict. Suppose the eyes are looking into the distance (Fig. 1 $A$, ignore the small white squares for now). If the head tilts sideways, right ear down, then the eyes counterroll around the same naso-

Received Aug. 23, 2000; revised Nov. 7, 2000; accepted Nov. 8, 2000.

This work was supported by Swiss National Science Foundation Grant 31-47287.96. D.T. is a Medical Research Council of Canada Scientist. We thank B. Disler and A. Züger for technical assistance, J. H. Cabungcal for participating in some of the experiments, and D. Angelaki, K. Beykirch, J. D. Crawford, S. Ferber, T. Haslwanter, D. Henriques, K. Hepp, K. Schreiber, D. Straumann, and T. Vilis for valuable comments on this manuscript.

Correspondence should be addressed to Dr. Hubert Misslisch, Department of Neurology, University of Zurich, Frauenklinikstrass 26, CH-8091 Zurich, Switzerland. E-mail: hubert.misslisch@nos.usz.ch.

Copyright (C) 2001 Society for Neuroscience $\quad 0270-6474 / 01 / 210001-05 \$ 15.00 / 0$ occipital axis, orthogonal to the plane of the paper in the figure (the real response is smaller, but we have magnified it in the drawing to make the geometry clear). Now suppose the eyes are converged (Fig. $1 B$ ). Were they now to counterroll, each rotating around the same naso-occipital axis of head tilt as before, they would no longer point at any common target. Their lines of sight would intersect nowhere. To avoid double vision, then, one motor system must suppress the other, or they might accommodate one another in some other way. For example, counterroll might alter its axes. If each eye rolled about its own line of sight, as in Figure $1 C$, rather than both eyes rolling about the naso-occipital axis, as in Figure $1 B$, then the two lines of sight would not break contact. We measured eye movements in three monkeys to test the interaction between vergence and two otolith reflexes, counterroll and a similar response called counterpitch.

\section{MATERIALS AND METHODS}

Subjects. Three juvenile rhesus monkeys (Macaca mulatta) were chronically prepared with skull bolts for head restraint. Dual search coils were implanted on both eyes under the conjunctiva (Hess, 1990). All procedures accorded with the NIH Guide for the Care and Use of Laboratory Animals and were approved by the Veterinary Office of the Canton of Zürich.

Recording and representation of binocular eye positions. Threedimensional eye positions were measured using magnetic search coils (Robinson, 1963) with an Eye Position Meter 3000 (Skalar, Delft, The Netherlands). Eye position was calibrated as described by Hess et al.

This article is published in The Journal of Neuroscience, Rapid Communications Section, which publishes brief, peerreviewed papers online, not in print. Rapid Communications are posted online approximately one month earlier than they would appear if printed. They are listed in the Table of Contents of the next open issue of JNeurosci. Cite this article as: JNeurosci, 2001, 21:RC126 (1-5). The publication date is the date of posting online at www.jneurosci.org.

http://www.jneurosci.org/cgi/content/full/4951 
A

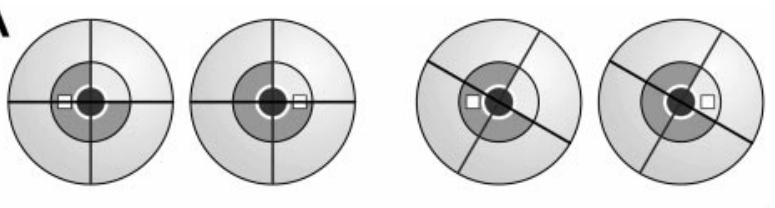

B
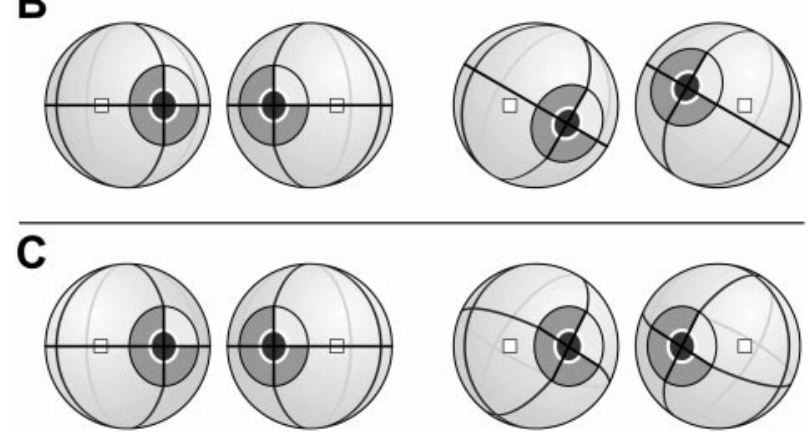

Figure 1. Why vergence and the otolith reflexes are in conflict. A, Two eyes look straight ahead. When the head tilts clockwise, or right ear down, the eyes counterroll about the same axis and in the opposite direction as the head, orthogonal to the plane of the paper. Note that the interocular axis is drawn horizontal in this and the following panels. $B$, The eyes converge to look at something close to the face. If the head now tilted clockwise, counterroll would again rotate both eyes about an axis orthogonal to the paper, driving apart the two lines of sight and causing double vision. Even when we look far away, counterroll complicates binocular vision. In $A$, the small squares are images cast on the two retinas by a single object; after counterroll, one image lies in the upper half of the retina and the other in the lower half, complicating the search by the brain for corresponding images in the two eyes. $C$, If the eyes counterrolled around their lines of sight instead of about the naso-occipital axis, then these lines would no longer miss each other, as they did in $B$, but the square images would still fall on opposite sides of the horizontal meridians of the retinas, introducing a vertical disparity.

(1992), digitized at a sampling rate of $833 \mathrm{~Hz}$, and stored on a computer for off-line data analysis. Eye positions were expressed as rotation vectors (Haustein, 1989), where the zero or reference positions were defined to be the orientations of the eye while the monkey fixated a target $0.8 \mathrm{~m}$ straight ahead.

Tasks. Monkeys sat in a primate chair mounted within an opaque sphere $1.6 \mathrm{~m}$ across. The monkey's head was restrained upright, with the lateral semicircular canals elevated $\sim 15^{\circ}$ anteriorly. Before the animal's face was a horizontal arc holding 13 light-emitting diodes (LEDs) spaced at $5^{\circ}$ intervals from $30^{\circ}$ left to $30^{\circ}$ right. The arc was so positioned and shaped, with a radius of $0.1 \mathrm{~m}$, that the monkey had to converge $17^{\circ}$ to fixate any one of the LEDs. The arc could be rotated into seven elevations ranging between $30^{\circ}$ down and $30^{\circ}$ up. Only one LED was lit at any one time, and animals were trained to fixate the illuminated LED. Quality of fixation was controlled with behavioral windows. Each experiment consisted of seven blocks, one for each elevation of the LED arc, and each block comprised 13 fixations, one for each LED in the arc, for a total of $7 \times 13=91$ fixations per experiment. Each LED was illuminated until the monkey fixated it for $1.5 \mathrm{sec}$. Throughout any one experiment, the monkey was stationary in a rolled or pitched position that ranged from $-100^{\circ}$ to $+100^{\circ}$. Data for $\pm 90^{\circ}$ roll and pitch orientations could be obtained only in one monkey (SU; see Fig. 3). Experiments were performed in light, i.e., with the background illumination on inside the opaque sphere. Orders of body positions, arc elevation, and LED illumination were randomized. Before each experiment, as a farviewing control, the opaque sphere was opened, and the animal made spontaneous eye movements in the light for $\sim 90 \mathrm{sec}$ looking at targets in the laboratory, all at least $1.5 \mathrm{~m}$ away.

\section{RESULTS}

When a monkey sat upright and looked around at distant objects, its ocular torsion (its rotation of the eye about the naso-occipital axis) was approximately zero, as marked by the gray line in Figure
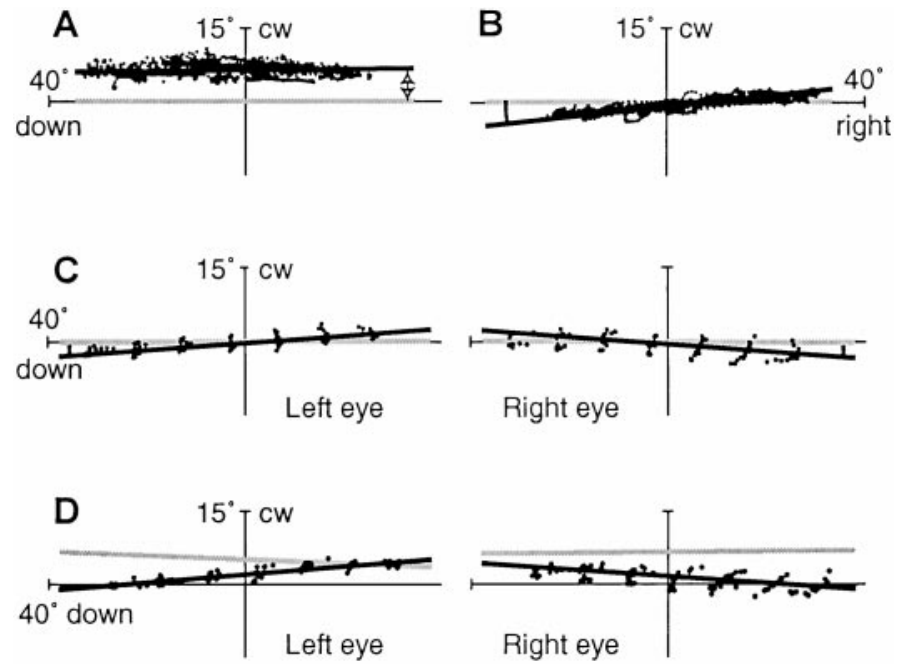

Figure 2. Otolith reflexes and vergence. $A$, When an upright monkey looks at distant targets, its ocular torsion stays near zero (gray line). When the monkey is tilted $60^{\circ}$ counterclockwise, or left ear down, its eye positions (black dots) are counterrolled $6.6^{\circ}$ clockwise (arrow). B, When the monkey is tilted vertically, $60^{\circ}$ nose down, it shows counterpitch; its ocular torsion varies as a function of horizontal eye position, so that the line of best fit tilts $5.0^{\circ}$. C, When a monkey sits upright and converges $17^{\circ}$, its ocular torsion varies as a function of vertical eye position, with opposite slopes in the two eyes. The lines of best fit (black lines) rotate $\sim 4^{\circ}$. During far viewing, ocular torsion is close to zero (gray lines). $D$, When a monkey is tilted $60^{\circ}$ left ear down, as in $A$, and converges $17^{\circ}$ to fixate near targets, its counterroll observed during far viewing (gray lines) is drastically reduced (black data points and lines).

$2 A$. When the monkey tilted $60^{\circ}$ counterclockwise, or left ear down, its eye positions (the black data points) were counterrolled, or shifted in the clockwise direction along the torsional axis (the ordinate). As the monkey looked around the laboratory, its eye position ranged over approximately $\pm 30^{\circ}$ both horizontally and vertically, but its ocular torsion remained approximately constant at 6 or $7^{\circ}$ (only the torsional and vertical components of eye position are shown here). We found the best-fit linear function relating torsional eye position to horizontal and vertical; from this function, we computed the torsional eye position when horizontal and vertical eye position were both zero (Figure $2 A$, the $y$-intercept of the black line). This torsional shift allows one to quantify changes in the torsional distribution of eye positions (Crawford and Vilis, 1991; Haslwanter et al., 1992; Mikhael et al., 1995; Bruno and van den Berg, 1997; Kapoula et al., 1999). In this case, the effect of counterroll was a torsional shift of $6.6^{\circ}$ clockwise.

Another otolith-ocular reflex, called counterpitch, operated when the monkey was tilted not sideways but vertically, $60^{\circ}$ nose down (Fig. $2 B$ ). Now the torsional component of eye position was not constant but instead varied as a function of the horizontal component. For example, when the monkey looked $30^{\circ}$ left, its eyes twisted some $2.5^{\circ}$ counterclockwise, and the twist reversed when it looked right (Crawford and Vilis, 1991; Haslwanter et al., 1992) (Why primates show this odd behavior we explain in Discussion.)

What are the torsional effects of vergence acting alone, in the absence of any otolith-ocular response to body tilt? We had the same monkey sit upright and converge its eyes $17^{\circ}$ to fixate a random-ordered series of 91 targets close to its face. Figure $2 C$ plots these 91 positions of the left and right eye, together with the best-fit linear functions. For both eyes, ocular torsion now varied 
with vertical eye position, although the relationships were opposite in the two eyes. We see, for instance, that the left eye twisted $\sim 2^{\circ}$ counterclockwise when it looked $30^{\circ}$ down and approximately the same distance clockwise when it looked $30^{\circ}$ up, whereas the right eye did the reverse. Neither eye showed any significant torsional shift. These are well-known features of human vergence (Mok et al., 1992; van Rijn and van den Berg, 1993; Minken and van Gisbergen, 1994; Mikhael et al., 1995; Bruno and van den Berg, 1997; Tweed, 1997; Somani et al., 1998; Kapoula et al., 1999; Steffen et al., 2000), here shown to apply to monkeys as well.

When the two systems were activated simultaneously, vergence overrode counterroll. Gray lines in Figure 2D show, again, the large torsional shifts caused by pure counterroll when the monkey was tilted $60^{\circ}$ counterclockwise while looking into the distance. Black lines and data points show the much smaller torsional shifts when the same monkey was tilted the same way while looking close.

All monkeys showed this suppression. To quantify the strength of the counterroll reflex during vergence, we measured the torsional shift, which we called $T_{\mathrm{cv}}$, that occurred when both systems were active and subtracted from this the very small torsional shift, called $T_{\mathrm{v}}$, that occurred when vergence alone was operating (example in Fig. $2 C$ ). This difference, $T_{\mathrm{cv}}-T_{\mathrm{v}}$, we compared with the torsional shift, $T_{\mathrm{c}}$, generated by pure counterroll (example in Fig. 2A). Gray symbols in Figure $3 A$ plot the amount of counterroll, $T_{\mathrm{c}}$, averaged across both eyes and all three monkeys, when they viewed far targets. For example, when the monkeys tilted $60^{\circ}$ clockwise, or right ear down, they counterrolled $5.5^{\circ}$ counterclockwise, on average. When the same monkeys viewed near targets their counterroll, $T_{\mathrm{cv}}-T_{\mathrm{v}}$, was reduced by $70 \%$. Statistically, this suppression of the counterroll reflex during vergence was highly significant (linear regression statistics, $T=-14 ; p<$ $0.0001)$.

Vergence also suppressed counterpitch. To quantify the counterpitch reflex, we again computed a best-fit linear function relating torsional eye position to horizontal and vertical. The angle of this function (Fig. $2 B$, black line) relative to the abscissa (the horizontal eye-position axis) is the usual measure of the strength of counterpitch (Crawford and Vilis, 1991; Haslwanter et al., 1992). We measured the angle between the line fitted during body tilt and the line in the control condition, with the monkey upright and looking far away. Quantified this way, the counterpitch angle was $4.6^{\circ}$, averaged across both eyes and all monkeys, when the animals were pitched $60^{\circ}$ nose up and viewed far targets (Fig. 3B, gray symbols). When these same animals viewed near targets, they no longer showed any systematic counterpitch as a function of body orientation (Fig. 3B, black symbols). Again, the suppression was highly significant (linear regression statistics, $T=-5.6 ; p<$ 0.0001).

Both of these interactions were one way. Vergence suppressed counterroll and counterpitch, but the otolith reflexes had no apparent influence on vergence. When monkeys viewed the near targets, they still showed the same $17^{\circ}$ vergence angle regardless of the orientation of the body with respect to gravity. Nor did body orientation affect the torsional dimension of vergence, the angle of the line relating torsional eye position to vertical (Fig. $2 C$ ). Regardless of body roll (circles) or pitch (squares), this angle stayed approximately constant at $\sim 3.6^{\circ}$ (Fig. $3 C$ ). Dividing this angle by the $17^{\circ}$ angle of vergence yields a ratio of 0.21 . It can be shown that the optimal ratio is 0.25 , in the sense that this value twists the eyes in a way that keeps the images of the visual plane
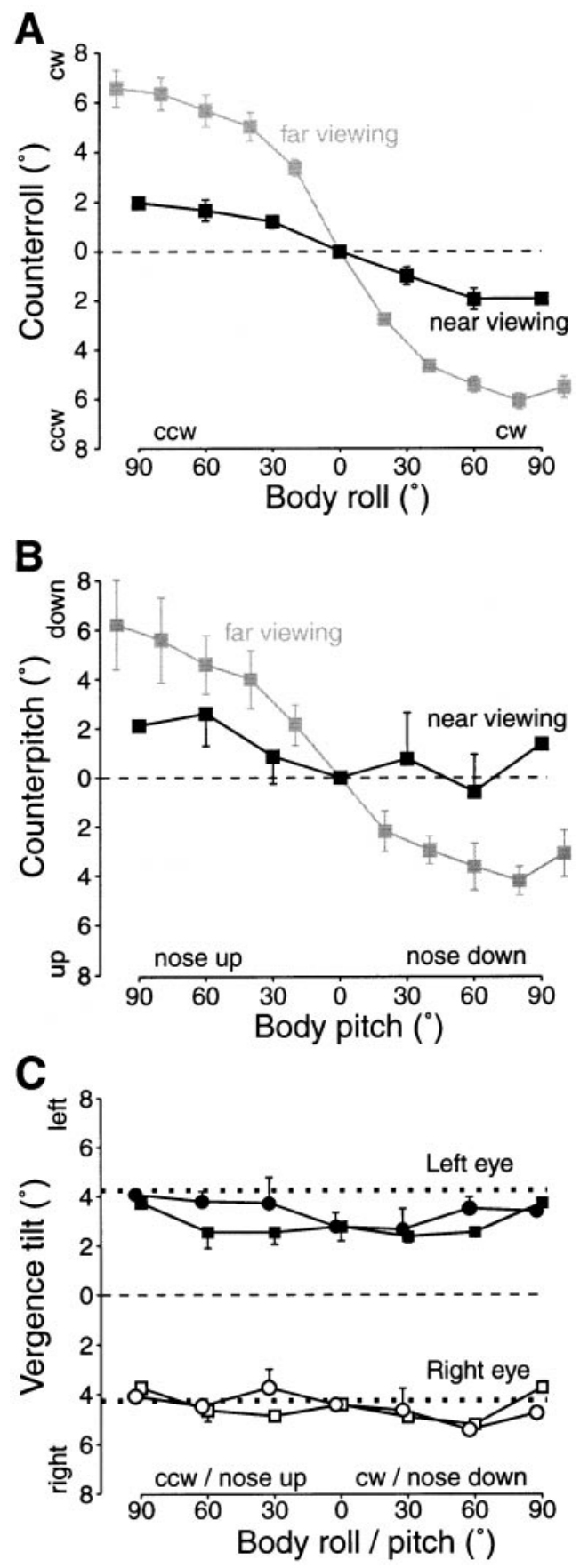

Figure 3. Vergence overrides otolith-ocular reflexes. A, Counterroll weakens by $\sim 70 \%$ when the eyes converge $17^{\circ}$. Data are averaged across both eyes and all three monkeys; error bars mark SEs. B, Counterpitch becomes weak and unsystematic when the eyes converge. $C$, Otolithocular reflexes do not alter the three-dimensional properties of vergence. The linear relationship between torsional and vertical eye position, plotted in Figure $2 C$, tilts through approximately the same angle regardless of body roll (circles) or pitch (squares). Error bars indicate one SE. Dotted lines mark the optimal tilt angles predicted by the visuomotor theory of binocular coordination (Tweed, 1997).

of the two retinas (the plane containing both lines of sight) perfectly aligned (van Rijn and van den Berg, 1993; Tweed, 1997). Thus, the ratio in monkeys is close to the theoretical optimal. Humans show ratios ranging between $\sim 0.17$ and the optimal value of 0.25 (Mok et al., 1992; van Rijn and van den Berg, 1993; 

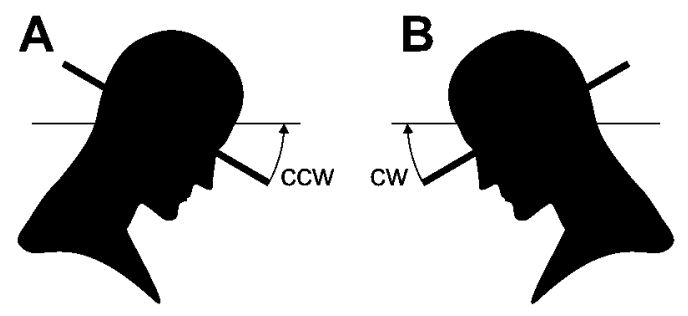

Figure 4. Counterpitch helps align the horizontal meridians of the eyes with the horizon. For explanation, see Discussion. $C C W$, Counterclockwise; $C W$, clockwise.

Minken and van Gisbergen, 1994; Mikhael et al., 1995; Bruno and van den Berg, 1997; Tweed, 1997; Somani et al., 1998; Kapoula et al., 1999; Steffen et al., 2000).

\section{DISCUSSION}

We have shown that there is a conflict between phylogenetically old gravity-driven reflexes and newer vergence mechanisms that serve stereopsis, and that vergence dominates. However, the old reflexes have not been eliminated. They coexist with the newer circuitry, balancing their conflicting functions. In lateral-eyed mammals, such as rodents and rabbits, the functions of counterroll and counterpitch seem clear; they help align the horizontal meridians of the retinas with the horizon when the animal rolls sideways or pitches nose up or nose down. What is the point of doing this? These animals have horizontally elongated foveae, or "visual streaks," so instead of our "lines of sight" they have "planes of sight." Counterroll and counterpitch help keep the planes of sight near the horizon plane, the better to watch for approaching danger (Carpenter, 1988).

We primates, on the other hand, can direct our gaze voluntarily, so we do not want reflexes that pin our eyes to the horizon. Only the torsional dimension of eye motion remains beyond our voluntary control, and it alone is still subject to gravity. For example, primate counterpitch, unlike rabbit counterpitch, does not raise or lower the eyes to point them at the horizon but instead twists them during rightward or leftward gaze. As we saw in Figure $2 B$, the eyes roll counterclockwise when they look left and clockwise when they look right, when the head is tipped nose down; when the head tips nose up, the pattern reverses, the eyes rolling clockwise on leftward gaze and counterclockwise on rightward gaze. Figure 4 explains how this strange pattern helps keep the horizontal meridians of the retinas aligned with the horizon. For example, when the head tips nose down, the horizon (thin line) tilts relative to the horizontal plane of the head (thick line). In Figure $4 A$, to simplify the geometry, we imagine that the subject is looking $90^{\circ}$ to his left. We view him from his right side, so we are looking in the same direction as he is, and therefore we see the horizon as he does: tilted counterclockwise relative to the horizontal plane of his head. This is the direction that counterpitch twists the eyes when a nose-down subject looks leftward (Fig. 2B), i.e., counterpitch brings the horizontal meridians of the retinas into better alignment with the horizon. In Figure $4 B$, the subject is looking $90^{\circ}$ to his right. We view him from his left side, so we see the horizon as he does, tilted clockwise, which is the direction that counterpitch twists the eyes when a nose-down subject looks rightward. Thus, the pattern of primate counterpitch is just what we would expect of a system that is trying to keep the horizontal meridians of the retinas horizontal but has control over just the torsional component of eye motion. Of course, the reflex is weak, as is primate counterroll, and this can be attributed to conflict with stereopsis.

We have seen that gravity-driven reflexes interfere with binocular vision when we converge. However, in a subtler way, these reflexes cause problems even when we are not converging. We see this in Figure $1 A$, where the small squares represent images cast on the two retinas by some single square object in front of the eyes. On the left side, the square falls to the right of the fovea in the right eye and to the left of the fovea in the left eye; that is, the two images are shifted along the horizontal meridian (this sort of horizontal disparity is the information the brain uses to compute how far away the object is). On the right side, because of counterroll, the two squares lie on opposite sides of the horizontal meridian, the square on the right retina lying below its meridian and the square on the left retina lying above. Hence, a vertical disparity has been introduced by the rotation of the eyes. It follows that the stereoptic system, when it looks for corresponding images in the two eyes, must search not just horizontally but also vertically, a two-dimensional task that increases the computational work. Reducing counterroll would reduce the vertical disparities, so presumably this is one reason the reflex has become so weak, even when the vergence angle is zero, in animals with stereopsis (Hunter, 1786; Nagel, 1868; Collewijn et al., 1985; Crawford and Vilis, 1991; Haslwanter et al., 1992).

Vertical disparities also explain why counterroll weakens during vergence rather than "adjusts" itself. That is, why does counterroll not adjust its axes when the eyes converge, turning the eyes around their lines of sight, as in Figure $1 C$, instead of around the same axis as the head roll, as in Figure $1 B$ ? That way, the lines of sight would still intersect at the fixation target. However, as Figure $1 C$ shows, a distant object would cast its images (the small squares) above the horizontal retinal meridian in one eye and below in the other, introducing a vertical disparity. Stereopsis is better served if counterroll is simply suppressed.

Counterroll was not completely eliminated by vergence but merely weakened by $\sim 70 \%$ (Fig. $3 A$ ). We believe that this degree of suppression is sufficient to allow binocular vision. One can compute that the vertical disparity on the fovea (approximately speaking, the vertical separation of the two lines of sight depicted schematically in Figure $1 B$ ) is approximately equal to the product of the counterroll angle and the vergence angle in radians, $d \approx$ $c \times v$. Figure $3 A$ shows that the counterroll angle was never larger than $\sim 2^{\circ}$ when vergence was $17^{\circ}$, which implies that vertical disparity reached $\sim 2 \times 17 \times \pi / 180=0.6^{\circ}$. When we measured vertical disparities directly during near target fixations, they averaged $0.64^{\circ}$ (SE of 0.05 ; all animals, all roll, and pitch body orientations). Stevenson and Schor (1997), using dynamic stereograms covering $12^{\circ}$ around the fovea, found that humans can tolerate vertical disparities of up to $\sim 0.75^{\circ}$, so these $0.64^{\circ}$ may be small enough for the visual system to deal with. Given the accuracy of binocular eye-coil recordings, however, we cannot rule out the possibility that the eyes verge very slightly in the vertical direction to reduce further this vertical disparity.

In summary, phylogenetically old, gravity-driven otolith reflexes try to keep the eyes level with the horizon. In primates, horizontal and vertical eye motion have come under voluntary control and are little influenced by the otolith reflexes. Torsional eye motion remains subject to gravity but is also controlled by circuits that serve binocular vision. The otolith reflexes and the newer stereoptic circuits call for incompatible eye movements, and the conflict intensifies when the eyes converge, resulting in the near-total suppression of the old reflexes. 


\section{REFERENCES}

Bruno P, van den Berg AV (1997) Relative orientation of primary positions of the two eyes. Vision Res 37:935-947.

Carpenter RHS (1988) Movements of the eyes, p 26. London: Pion.

Collewijn H, van der Steen J, Ferman L, Jansen TC (1985) Human ocular counterroll: assessment of static and dynamic properties from electromagnetic scleral coil recordings. Exp Brain Res 59:185-196.

Crawford JD, Vilis T (1991) Axes of eye rotation and Listing's law during rotations of the head. J Neurophysiol 65:407-423.

Fleisch A (1922) Tonische Labyrinthreflexe auf die Augenstellung. Pflügers Arch 194:554-573.

Haslwanter T, Straumann D, Hess BJM, Henn V (1992) Static roll and pitch in the monkey: shift and rotation of Listing's plane. Vision Res 32:1341-1348.

Haustein W (1989) Considerations on Listing's Law and the primary position by means of a matrix description of eye position control. Biol Cybern 60:411-420.

Hess BJM (1990) Dual-search coil for measuring 3-dimensional eye movements in experimental animals. Vision Res 30:597-602.

Hess BJM, van Opstal AJ, Straumann D, Hepp K (1992) Calibration of three-dimensional eye position using search coil signals in the rhesus monkey. Vision Res 32:1647-1654.

Hughes A (1972) Vergence in the cat. Vision Res 12:1961-1994.

Hunter J (1786) Observations on certain parts of the animal oeconomy. London.

Kapoula Z, Bernotas M, Haslwanter T (1999) Listing's plane rotation with convergence: role of disparity, accommodation, and depth perception. Exp Brain Res 126:175-186.

Mikhael S, Nicolle D, Vilis T (1995) Rotation of Listing's plane by horizontal, vertical and oblique prism-induced vergence. Vision Res 35:3243-3254.
Minken AWH, van Gisbergen JAM (1994) A three-dimensional analysis of vergence movements at various levels of elevation. Exp Brain Res 101:331-345

Mok D, Ro A, Cadera W, Crawford JD, Vilis T (1992) Rotation of Listing's plane during vergence. Vision Res 32:2055-2064.

Nagel A (1868) Ueber das Vorkommen von wahren Rollungen des Auges um die Gesichtslinie. Arch f Ophth 14:228-246.

Robinson DA (1963) A method of measuring eye movement using a scleral search coil in a magnetic field. IEEE Trans Bio-Med Electron 10:137-145.

Somani RA, DeSouza JF, Tweed D, Vilis T (1998) Visual test of Listing's law during vergence. Vision Res 38:911-923.

Steffen H, Walker MF, Zee DS (2000) Rotation of Listing's plane with convergence: independence from eye position. Invest Ophthalmol Vis Sci 41:715-721.

Stevenson SB, Schor CM (1997) Human stereo matching is not restricted to epipolar lines. Vision Res 37:2717-2723.

Tweed D (1997) Visual-motor optimization in binocular control. Vision Res 37:1939-1951.

van der Hoeve J, de Kleijn A (1917) Tonische Labyrinthreflexe auf die Augen. Pflügers Arch 169:241-262.

van Rijn LJ, van den Berg AV (1993) Binocular eye orientation during fixations: Listing's law extended to include eye vergence. Vision Res 33:691-708.

von Helmholtz H (1867) Handbuch der physiologischen Optik, Ed 1, Vol 3. Reprint (Southall JPC, translator) as Treatise on physiological optics. Rochester, NY: Optical Society of America, 1925.

Wheatstone C (1838) Contributions to the physiology of vision. I. On some remarkable, and hitherto unobserved, phenomena of binocular vision. Philos Trans R Soc Lond B Biol Sci 2:371-393.

Zuidam I, Collewijn H (1979) Vergence eye movements of the rabbit in visuomotor behavior. Vision Res 19:185-194. 